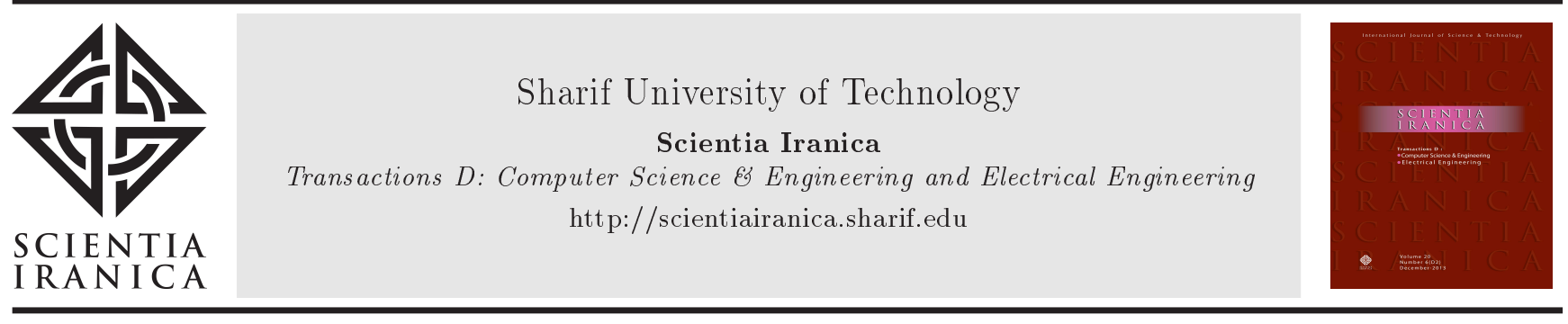

\title{
Adaptive sliding mode control for multi-machine power systems under normal and faulted conditions
}

\author{
A. Samanfar ${ }^{\mathrm{a}}$, M.R. Shakarami ${ }^{\mathrm{a}, *}$, J. Soltani Zamani ${ }^{\mathrm{b}}$, and E. Rokrok ${ }^{\mathrm{a}}$ \\ a. Department of Electrical Engineering, Lorestan University, Lorestan, Khorramabad, Iran. \\ b. Department of Electrical Engineering, Islamic Azad University, Khomeinishahr Branch, Khomeinishahr, Iran. \\ Received 29 March 2020; received in revised form 12 September 2020; accepted 28 December 2020
}

\author{
KEYWORDS \\ Adaptive sliding mode \\ control; \\ Power system \\ stability; \\ Lyapunov theory; \\ sequence networks; \\ Space phasor.
}

\begin{abstract}
This paper proposes a new Adaptive Sliding Mode (ASM) decentralized excitation controller to improve the stability of multi-machine power systems under different perturbations such as system's parametric and structural uncertainties. The stability of the closed-loop system is proved by the Lyapunov stability theory. The proposed controller is evaluated through simulation on the standard IEEE 33-bus-bar power system which contains 6 synchronous machines and an HVDC link. The simulation results indicate good robustness and satisfactory performance of the proposed controller. Moreover, this study employs the method of space-phasor-based sequence networks, which is a procedure for the dynamic analysis of modern power systems under transient asymmetrical faults. The method considers the complete dynamics of synchronous machines and the HVDC link and provides the possibility of taking into account the sequence networks dynamics.

(C) 2022 Sharif University of Technology. All rights reserved.
\end{abstract}

\section{Introduction}

Given that power demand grows rapidly and the expansion of transmission and generation is restricted by the limited availability of resources and the strict environmental constraints, power systems are much more loaded today than ever. This issue causes power systems to operate at a level close to their stability limits [1]. In order to enhance the stability of power systems and maintain their synchrony, a wide range of controllers have been introduced in the power system literature to generate a supplementary control signal for the synchronous machine excitation system [2], FACTS devices [3], HVDC link [4], and renewable

\footnotetext{
*. Corresponding author.

E-mail addresses: Samanfar.am@fe.lu.ac.ir and

a.samanfar@gmail.com (A. Samanfar);

Shakarami.mr@lu.ac.ir (M.R.Shakarami);

jsoltani@iaukhsh.ac.ir (J. Soltani Zamani);

Rokrok.e@lu.ac.ir (E.Rokrok)
}

energy plants [5]. Among these, the synchronous machine excitation system controllers are known as flexible and economical ways to improve power system stability [2]. Various methods have been used for designing excitation system controllers. Linear Control (LC) techniques are commonly used in designing classical controllers that ensure the asymptotic stability of the operation point under small perturbations $[6,7]$. A number of techniques in scientific resources have been developed to improve the performance of classical controllers $[8,9]$. In practice, the efficiency of the controllers may be affected due to many variations in the operation point and the nonlinear behavior of the power systems.

In order to consider the complete operation region and deal with the nonlinear behavior of the power systems, nonlinear controllers have been implemented by power systems studies. The direct Lyapunov method [10] and feedback linearization [11] are the most common nonlinear controllers in power systems. These methods can solve the problems of LC techniques, but they are not robust enough in the presence of system 
uncertainties. Neural networks [12] and fuzzy logic [13], as intelligent control techniques, can avoid the former limitations. These are powerful methods and can help to improve the stability of the power system in different operation conditions. However, these methods are time-consuming which limits their use in practice. Also, in the neural network-based controllers, much training data are needed, which is difficult to obtain from the physical power systems.

The Sliding Mode Control (SMC) is a wellknown technique to improve the stability of nonlinear systems under parametric uncertainties and external disturbances [14-21]. In recent years, several Power System Stabilizers (PSS) based on SMC scheme have been proposed in the literature [16-21]. In these controllers, the control laws with fixed control gains have been used. Since the upper bound of the system's uncertainties may not be easily obtained, a large quantity for this parameter is assumed to guarantee the stability of the system. This may lead to undesired chattering and a very high control input power [22]. Also, most of these controllers require not only the local generator information but also the power system information which limits their applicability to large power systems. Moreover, these controllers have been evaluated just in balanced conditions, while the probability of unbalanced conditions and in particular asymmetrical faults is high in power systems [18-22].

The main contribution of this paper is to propose an Adaptive Sliding Mode (ASM) decentralized excitation controller to improve the stability of modern power systems. The proposed controller has an adaptive control gain such that despite the Conventional Sliding Mode (CSM) controller, its initial setting should be smaller than the upper bound of the system uncertainties. This feature eliminates the need for determining the upper bound of the system uncertainties, which is difficult to obtain in practice. Furthermore, the adaptive control gain could reduce the probability of the occurrence of chattering phenomenon and also, reduce the control costs [23]. It is necessary to note that the proposed decentralized controller requires only local generator information, which is a merit for large power systems. The proposed controller does not need observers and it considers the neglected dynamics as internal or external perturbation. In this study, the proposed controller has been evaluated for symmetrical and asymmetrical faults. The dynamic analysis of faulty systems is performed using the method of space-phasor-based sequence networks. The method considers complete dynamics of synchronous machines, HVDC links, and sequence networks.

\section{ASM controller design}

Here, an ASM controller is designed to improve the stability of the practical multi-machine modern power systems. In this study, a local controller is designed for each synchronous machine and the effect of other synchronous machines is considered as a perturbation by means of power oscillations. Thus, the communication among the controllers is not needed [21]. For designing the ASM controller, the third-order model of the synchronous machine [1] is used:

$$
\begin{aligned}
& \frac{d \delta}{d t}=\omega_{r}-\omega_{0}, \\
& \frac{2 H}{\omega_{0}} \frac{d \omega_{r}}{d t}=T_{L}-T_{e}-D \cdot\left(\omega_{r}-\omega_{0}\right), \\
& \frac{d E_{q}^{\prime}}{d t}=\frac{1}{T_{d o}^{\prime}}\left[E_{f d}-E_{q}^{\prime}-\left(X_{d}-X_{d}^{\prime}\right) \cdot i_{d s}^{r}\right] .
\end{aligned}
$$

Moreover, the electrical torque is:

$$
T_{e}=E_{q}^{\prime} \cdot i_{q s}^{r}+\left(X_{q}-X_{d}^{\prime}\right) \cdot i_{q s}^{r} \cdot i_{d s}^{r} .
$$

Due to the slow variations of $i_{q s}^{r}$ and $i_{d s}^{r}$, the derivations of these currents are assumed to be zero. Hence, derivation of $T_{e}$ with respect to time gives:

$$
\frac{d T_{e}}{d t} \cong \frac{d E_{q}^{\prime}}{d t} \cdot i_{q s}^{r}
$$

Substituting Eq. (3) into Eq. (5) and defining $B=\frac{1}{T_{d o}^{\prime}}$ lead to the following:

$$
\frac{d T_{e}}{d t}=i_{q s}^{r} . B\left[E_{f d}-E_{q}^{\prime}-\left(X_{d}-X_{d}^{\prime}\right) \cdot i_{d s}^{r}\right] .
$$

The nominal values of the parameters in Eq. (6) can be defined as follows:

$$
B_{n}, X_{d-n}, X_{d-n}^{\prime} \text {. }
$$

The equations between the real and nominal values of these parameters are considered as follows:

$$
\begin{aligned}
& B=B_{n}+\Delta B, \\
& X_{d}=X_{d-n}+\Delta X_{d}, \\
& X_{d}^{\prime}=X_{d-n}^{\prime}+\Delta X_{d}^{\prime} .
\end{aligned}
$$

Substituting Eqs. (8), (9), and (10) into Eqs. (4) and (6) gives:

$$
\begin{aligned}
& T_{e}=E_{q}^{\prime} \cdot i_{q s}^{r}+\left(X_{q-n}-X_{d-n}^{\prime}\right) \cdot i_{q s}^{r} \cdot i_{d s}^{r}+\zeta_{1}, \\
& \frac{d T_{e}}{d t}=i_{q s}^{r} \cdot B_{n}\left[E_{f d}-E_{q}^{\prime}-\left(X_{d-n}-X_{d-n}^{\prime}\right) \cdot i_{d s}^{r}\right]+\zeta_{2},
\end{aligned}
$$

where $\zeta_{1}$ and $\zeta_{2}$ as the model uncertain functions can be expressed as:

$$
\begin{aligned}
\zeta_{1}= & \left(\Delta X_{q}-\Delta X_{d}^{\prime}\right) \cdot i_{d s}^{r} i_{q s}^{r}, \\
\zeta_{2}= & i_{q s}^{r} \cdot \Delta B\left[E_{f d}-E_{q}^{\prime}-\left(X_{d}-X_{d}^{\prime}\right) \cdot i_{d s}^{r}\right] \\
& \quad-i_{q s}^{r} \cdot B_{n}\left[\left(\Delta X_{d}-\Delta X_{d}^{\prime}\right) \cdot i_{d s}^{r}\right] .
\end{aligned}
$$

Since all the parameters in Eqs. (13) and (14) are 
bounded, it can be concluded that $\zeta_{1}$ and $\zeta_{2}$ are bounded functions. To design the proposed ASM controller, the electrical torque and the mechanical speed error variables are defined as:

$$
e_{T}=T_{e}^{*}-T_{e}, \quad e_{\omega}=\omega_{r}-\omega_{0},
$$

where these errors represent the difference between the real and reference values. The output speed error $e_{\omega}$ can be eliminated by definition and the reference value of the electrical torque $T_{e}^{*}$ is as follows:

$$
T_{e}^{*}=K_{e} \int\left(\omega_{r}-\omega_{0}\right) \cdot d t
$$

where $K_{e}$ is a positive scalar. At first, the controller is designed by considering the nominal values of the model and neglecting the model uncertain functions $\left(\zeta_{1}\right.$ and $\zeta_{2}$ ). The sliding surface is selected as follows:

$$
S=e_{T}+e_{\omega}+K \int\left(e_{T}+e_{\omega}\right) \cdot d t
$$

where $K$ is a positive scalar. In accordance with the sliding mode technique, the derivation of the sliding surface is set to zero:

$$
\dot{S}=0 \rightarrow \dot{e}_{T}+\dot{e}_{\omega}+K\left(e_{T}+e_{\omega}\right)=0,
$$

or:

$$
\dot{S}=M+N=0,
$$

where:

$$
M=\dot{e}_{T}+K e_{T}, \quad N=\dot{e}_{\omega}+K e_{\omega} .
$$

It is claimed that by setting $M$ to zero, $N$ will also be zero:

$$
M=\dot{e}_{T}+K e_{T}=0 \underset{t \rightarrow \infty}{\stackrel{K>0}{\longrightarrow}} e_{T}=0, \quad \dot{e}_{T}=0 .
$$

as:

$$
\dot{e}_{T}=0 \rightarrow \dot{T}_{e}^{*}-\dot{T}_{e}=0 \rightarrow K_{e}\left(\omega_{r}-\omega_{0}\right)-\dot{T}_{e}=0 .
$$

From Eq. (2) and assuming that $T_{L}$ is a constant, one can derive that:

$$
\dot{T}_{e}=-\frac{2 H}{\omega_{0}} \ddot{e}_{\omega}-D \dot{e}_{\omega} .
$$

Substituting Eq. (23) in Eq. (22) gives:

$$
\begin{aligned}
& \frac{2 H}{\omega_{0}} \ddot{e}_{\omega}+D \dot{e}_{\omega}+K_{e} e_{\omega}=0, \frac{\frac{2 H}{\omega_{0}}, D, K_{e}>0}{t \rightarrow \infty} \\
& e_{\omega}=0, \quad \dot{e}_{\omega}=0, \ddot{e}_{\omega}=0 \quad \Rightarrow N=0 .
\end{aligned}
$$

In doing so, the claim is proven. Thus, in order to obtain the equivalent ASM controller, only $M$ is considered here. Combination of Eqs. (15), (16), and (20) would lead to the following:

$$
M=K_{e} e_{\omega}-\dot{T}_{e}+K e_{T} .
$$

Setting Eq. (25) to zero and replacing Eq. (6) in it achieve the control law as follows:

$$
\begin{aligned}
E_{f d-n}= & E_{q}^{\prime}+\left(X_{d-n}-X_{d-n}^{\prime}\right) i_{d s}^{r}+\frac{K_{e}}{i_{q s}^{r} \cdot B_{n}} e_{\omega} \\
& +\frac{K}{i_{q s}^{r} \cdot B_{n}} e_{T} .
\end{aligned}
$$

In Eq. (26), the parameters take nominal values. If the model parameters are not equal to the nominal values, system responses will not settle on the sliding surface. By considering Eq. (19) and substituting $M$ and $N$ into it, we can have:

$$
\dot{S}=M+N=K_{e} e_{\omega}-\dot{T}_{e}+K e_{T}+\dot{e}_{\omega}+K e_{\omega} .
$$

In the presence of uncertainties, according to Eqs. (11) and (12), $\dot{T}_{e}$ and $T_{e}$ contain $\zeta_{1}$ and $\zeta_{2}$ as uncertainty terms, respectively. Thus, the derivation of the sliding surface is defined as follows:

$$
\dot{S}=\dot{S}_{n}+\eta,
$$

where $\dot{S}_{n}$ is the derivation of the sliding surface for the nominal values and $\eta$ is the system uncertain function. $\dot{S}_{n}$ and $\eta$ are obtained as follows:

$$
\begin{aligned}
& \dot{S}_{n}=M=K_{e} \cdot e_{\omega}-\dot{T}_{e}+K \cdot e_{T}, \\
& \eta=-\zeta_{1}-\zeta_{2}+N=-\zeta_{1}-\zeta_{2}+\dot{e}_{\omega}+K e_{\omega} .
\end{aligned}
$$

In this paper, the modified control law is proposed to be:

$$
E_{f d}=E_{f d-n}+\tilde{E}_{f d},
$$

where $E_{f d-n}$ is the same as Eq. (26) used for nominal conditions. Also, the adaptive term $\tilde{E}_{f d}$ is modified below to deal with the bounded uncertainties expressed in Eq. (30):

$$
\tilde{E}_{f d}=\frac{1}{i_{q s}^{r} \cdot B_{n}}\left(K_{C} S+\Gamma \operatorname{sgn}(S)\right),
$$

where $\Gamma$ is an adjustable gain constant. The desired value of this parameter is called $\Gamma_{d}$ and must satisfy the following condition:

$$
|\eta|<\Gamma_{d} .
$$

The adaptive law for adjusting $\Gamma$ is:

$$
\dot{\Gamma}=\frac{1}{\alpha}|S| \text {. }
$$

In Eq. (34), $\alpha$ is a positive scalar called adaption gain. The adaption speed can be tuned by $\alpha$. Also, choosing 
a suitable adaption gain $\alpha$ can effectively avoid high control activity in the reaching mode [23]. It can be proven that the proposed controller makes the closedloop system stable in the presence of uncertainties and disturbances, and the system response will certainly be on the sliding surface. In this regard, $\dot{S}$ can be simplified in the following by combining Eqs. (28)-(32) and some mathematical manipulations:

$$
\dot{S}=-\left(K_{C} S+\Gamma \operatorname{sgn}(S)\right)+\eta .
$$

In order to examine the validity of the proposed control law, the following Lyapunov function is defined as:

$$
V=\frac{1}{2} S^{2}+\frac{1}{2} \alpha \tilde{\Gamma}^{2}
$$

where $\tilde{\Gamma}=\Gamma-\Gamma_{d}$. By taking the time derivative of $V$, one can obtain the following:

$$
\dot{V}=S \dot{S}+\alpha \tilde{\Gamma} \dot{\tilde{\Gamma}}
$$

By substituting Eq. (35) into Eq. (37) and considering $\dot{\tilde{\Gamma}}=\dot{\Gamma}$, Eq. (37) becomes:

$$
\dot{V}=S\left(-K_{C} S-\Gamma \operatorname{sgn}(S)+\eta\right)+\left(\Gamma-\Gamma_{d}\right) S \operatorname{sgn}(S) .
$$

Finally, according to $\rightarrow S \cdot \operatorname{sgn}(S)=|S|$, the derivation of Lyapunov function can be written as follows:

$$
\dot{V}=-K_{C} S^{2}+\eta S-\Gamma_{d}|S| .
$$

Based on Ineq. (33), one can derive $\eta S<\Gamma_{d}|S|$ and then, reach:

$$
\dot{V}<0
$$

Hence, the derivation of the Lyapunov function is a negative function. Therefore, the convergence of $S$ and $\tilde{\Gamma}$ is guaranteed and both of them reach zero in finite time. This completes the proof.

\section{Power system dynamic analysis}

In [24], a method based on space phasors was introduced for dynamic analysis of transient symmetrical or asymmetrical faults in power systems. Upon developing the method described in [24], this section discusses the dynamic analysis of a transient SingleLine-to-Ground (SLG) fault in the IEEE 33-bus standard network including an HVDC link. The method described here can easily be implemented to analyze other symmetrical and asymmetrical series or shunt faults. Figure 1 shows the IEEE 33-bus standard network.

This network consists of three areas: 33 buses, 6 synchronous machines, and an HVDC link. Figure 2 depicts the HVDC general circuit and the proposed

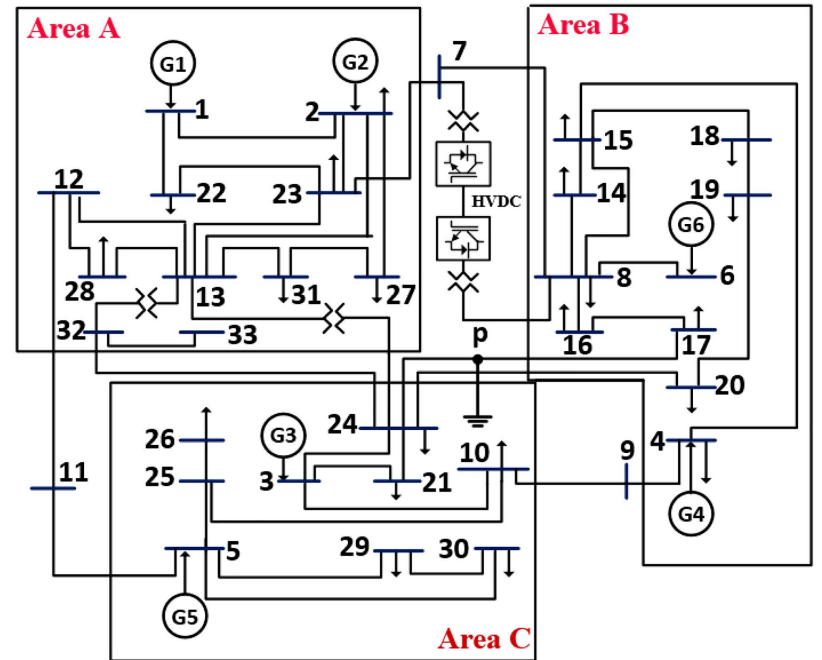

Figure 1. IEEE 33-bus standard network with an HVDC link.
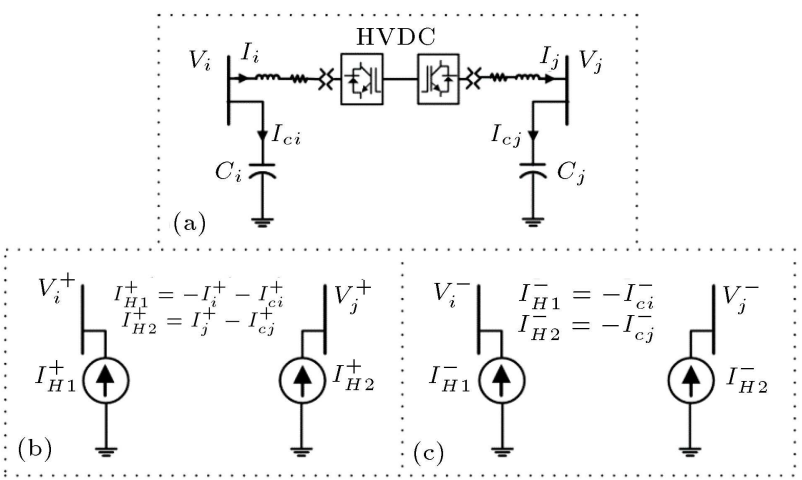

Figure 2. HVDC model: (a) Schematic (b) in positive-sequence network, and (c) in negative-sequence network.

models for an HVDC link in positive and negative SNs. Thus, an HVDC link can be modeled in the sequence networks by means of its positive- and negativesequence current space phasors.

Before the occurrence of a fault, the network equilibrium point is obtained through the NewtonRaphson AC load flow. When an SLG fault occurs at point "P" (between buses 17 and 24), a virtual bus is considered at this point with the number 34 . Figure 3 shows the sequence networks of the system.

In this figure, PSN, NSN, and ZSN expressions indicate the positive-, negative-, and zero-sequence networks, respectively. Then, the bus-impedance matrices of the positive-, negative-, and zero-sequence networks with the titles $Z^{+}, Z^{-}$, and $Z^{0}$ are composed. The dimensions of these matrices are $34 \times 34$ in this example. In order to calculate the fault-current sequence components, the equivalent circuits of the sequence networks are configured at " $\mathrm{P}$ ".

The type of fault determines how to connect the sequence networks at point "P". Figure 4 illustrates 

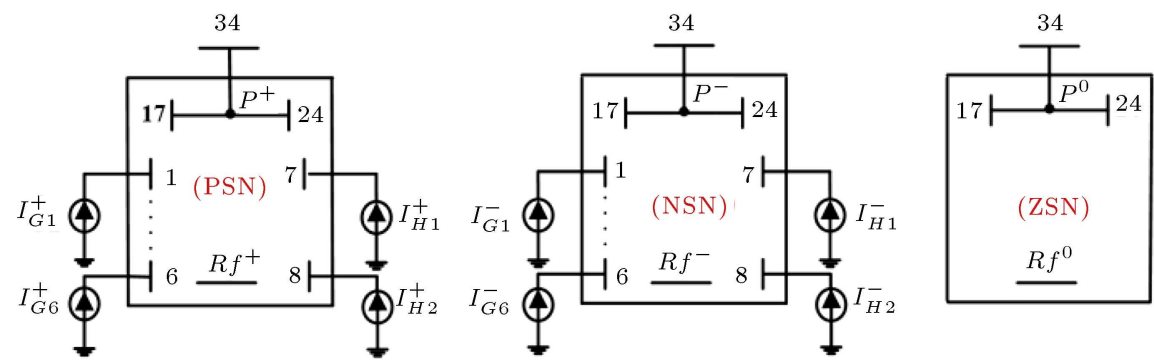

Figure 3. The sequence networks of Figure 1. PSN: Positive Sequence Network, NSN: Negative Sequence Network, ZSN: Zero Sequence Network.

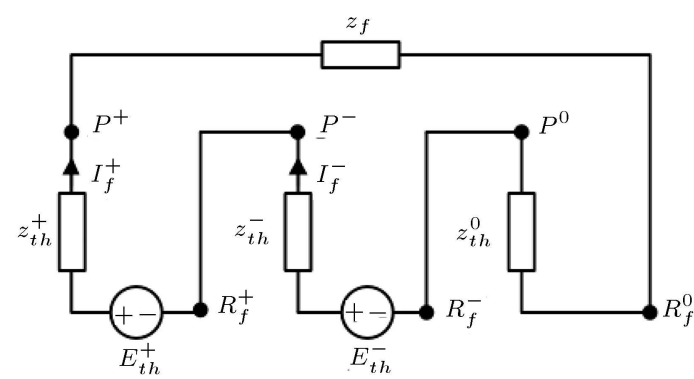

Figure 4. Connecting sequence networks for an SLG fault.

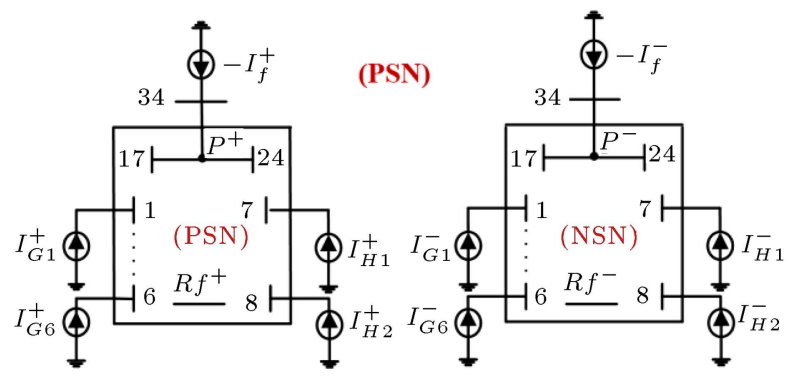

Figure 5. Modelling the fault current sequence components in PSN (Positive-Sequence Network) and NSN (Negative-Sequence Network).

how to connect these equivalent circuits for an SLG fault.

According to Figure 4, the fault current sequence components are as follows:

$$
I_{f}^{+}=I_{f}^{-}=I_{f}^{0}=\frac{E_{t h}^{+}+E_{t h}^{-}}{\left(z_{t h}^{+}+z_{t h}^{-}+z_{t h}^{0}+z_{f}\right)} .
$$

In Eq. (41), quantities with superscripts (+), $(-)$, and (0) denote the positive-, negative-, and zerosequence quantities, respectively. $I_{f}$ is the fault current and $z_{f}$ is the impedance of the fault. After calculating the fault-current sequence components, each component is injected as an injection-current source into the "P" in the corresponding sequence network. Figure 5 shows how to model the fault current in positive- and negative-sequence networks.

The fault current sequence components are considered in the current vectors of positive- and negative- sequence networks. Then, the terminal sequence voltages $\left(V_{t}^{+}, V_{t}^{-}\right)$of the synchronous machines in different sequence networks are obtained through the quasistatic Y-matrix equations of sequence networks. These voltages are in the synchronous reference frame and must be transferred to the rotor reference frame according to transform functions as follows:

$$
\begin{aligned}
& F_{t}^{r+}=F_{t}^{+} e^{-j \delta}, \\
& F_{t}^{r-}=F_{t}^{-} e^{-j\left(2 \omega_{e} t+\delta\right)} .
\end{aligned}
$$

In these equations, $F_{t}^{+}$and $F_{t}^{-}$are the electrical variables (voltage or current) of the synchronous machines in the synchronous reference frame, respectively, and $F_{t}^{r+}$ and $F_{t}^{r-}$ are those variables in the rotor reference frame. In Eqs. (42) and (43), $\delta$ is the angle between the quadrature axes of the rotor and the synchronous reference frames. These equations guarantee eliminating time-varying inductances in the Park's voltage equations in unbalanced conditions. Therefore, the conventional full-order differential equations of the synchronous machine in the rotor reference frame could be used in unbalanced conditions [24]. In the next section, the procedure of this method is presented.

\section{Dynamic analysis procedure}

In the previous section, a method for obtaining the mathematical model of the power system during fault time was developed. Here, the steps of the procedure for the dynamic analysis of a faulted power system are presented as follows:

1. Solve the power system load-flow equations to get the system's initial values;

2. Form $[\mathrm{Y}]$ matrices of the three-sequence networks;

3. According to the fault type, expand and modify the [Y] matrix of the sequence networks to include the fictitious bus;

4. Inject the current space phasors of synchronous machines and HVDC link at their related bus or buses into each sequence network; 
5. Determine the Thevenin equivalent circuits seen from the fault point and then, calculate the faultcurrent space phasors;

6. Separate the sequence networks and inject the current sequence components of the synchronous machines, HVDC link, and fault in their related buses into each sequence network (Figure 5);

7. Solve the Y-matrix equations of positive- and negative-sequence networks to obtain the terminal sequence voltages of synchronous machines and HVDC link;

8. For each synchronous machine, transfer the values of the terminal sequence voltages to its rotor reference frame (Eqs. (42) and (43)). Then, solve the differential equations of the synchronous machine and update the values of its state variables. By using the updated state variables, calculate the outputcurrent sequence components of the synchronous machine and transfer them to the synchronous reference frame through Eqs. (42) and (43);

9. For the HVDC link, solve the differential equations and calculate its injection currents at both ends in the positive- and negative-sequence networks;

10. If the fault is cleared, print the results; otherwise, go to Step 4.

Based on the above procedure, a MATLAB code program was developed. The program uses the numerical fourth-order static Range-Kutta method with a time step $(\Delta t)$ of $10^{-4}$ to solve nonlinear equations of synchronous machines and the HVDC link. The program can be used for the dynamic analysis of power systems facing any kind of shunt faults.

\section{Simulation results}

Here, the proposed ASM controller is tested on the IEEE standard 33-bus network (Figure 1) including the full-order model of synchronous machines and complete dynamics of the HVDC link. For synchronous machines, two damper windings on the $q$-axis and a damper winding on the $d$-axis were considered [25]. Also, the HVDC link is fully modeled such that it includes its filters and switching circuits. In this study, network loads, transmission lines, power transformers, and fault are modeled as constant impedances. The machine specifications, the HVDC link parameters, and the network load-flow data were provided in $[3,26]$, and IEEE website, respectively. In this network, it is supposed that the excitation systems of G2-G6 are equipped with the proposed ASM controller. In the following, the proposed controller is evaluated by performing different scenarios in the network.

\section{A. Scenario 1}

In this scenario, the performance of the proposed ASM controller is evaluated by using it in the IEEE 33-bus network. It is assumed that before $t=0 \mathrm{sec}$, the HVDC link is not in service and the network has been in a steady state condition. Then, the following events are applied in the network at $t=0 \mathrm{sec}$ :

1. An initial error of $+20 \%$ is considered in the reactance of the transmission lines 1-22 and 2-23.

2. An initial error of $-20 \%$ is considered in the direct axis transient reactance of the synchronous machines G3-G6.

Figure 6 shows the rotor angles of synchronous machines before installing controllers in the network.

In order to cope with the mentioned uncertainties and to achieve the minimum network stabilizing time, G2-G6 synchronous machines are equipped with the proposed ASM controller. The controllers' parameters are obtained through trial and error. Figures 7 and 8

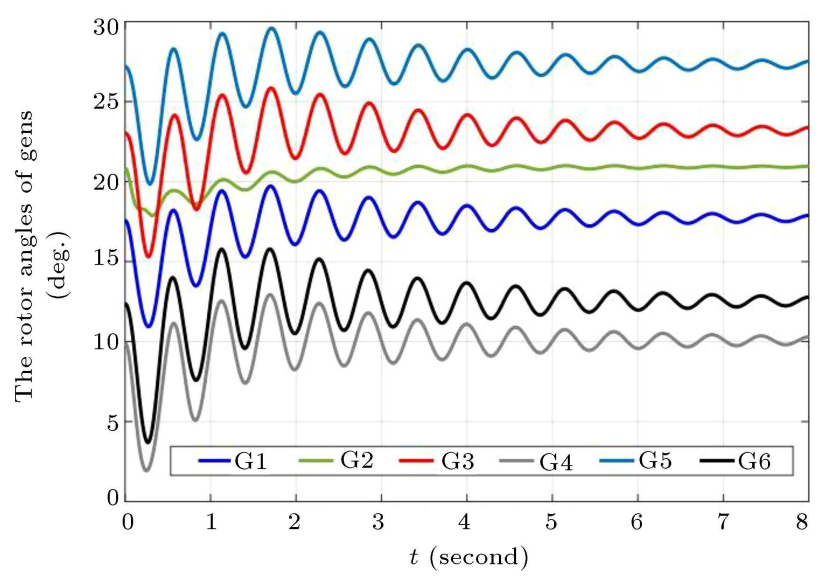

Figure 6. Rotor angles of the synchronous machines without controllers in Scenario 1.

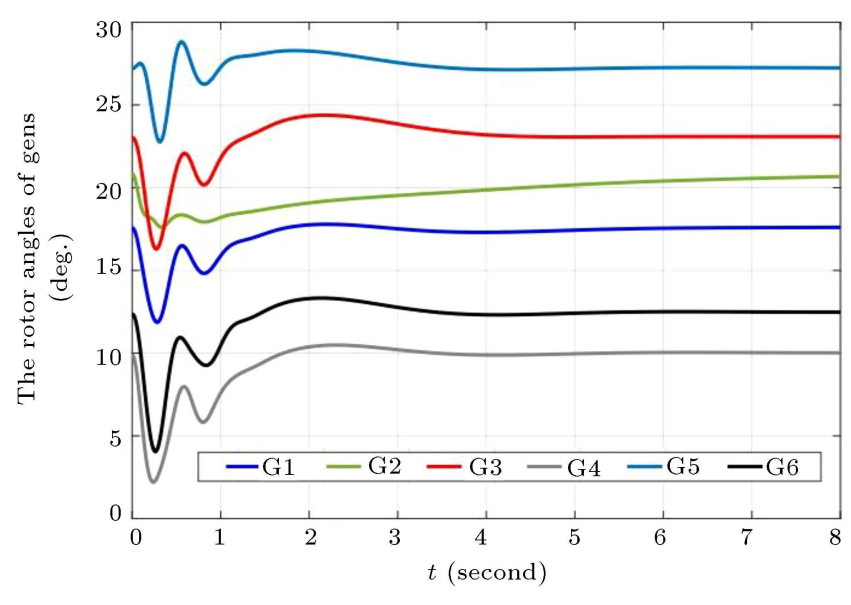

Figure 7. Rotor angles of the synchronous machines with ASM Controllers in Scenario 1. 
show the rotor angles and rotor angular speeds of the synchronous machines, respectively, after installing the proposed ASM controllers on G2-G6.

As shown in these figures, the rotor angles and the angular speeds of all synchronous machines reach their reference values in finite time despite the uncertainties. Figure 9 illustrates the variation of the adjustable gain for the ASM controllers in this study. A small positive initial value (0.001) was chosen for $\Gamma$ in all controllers.

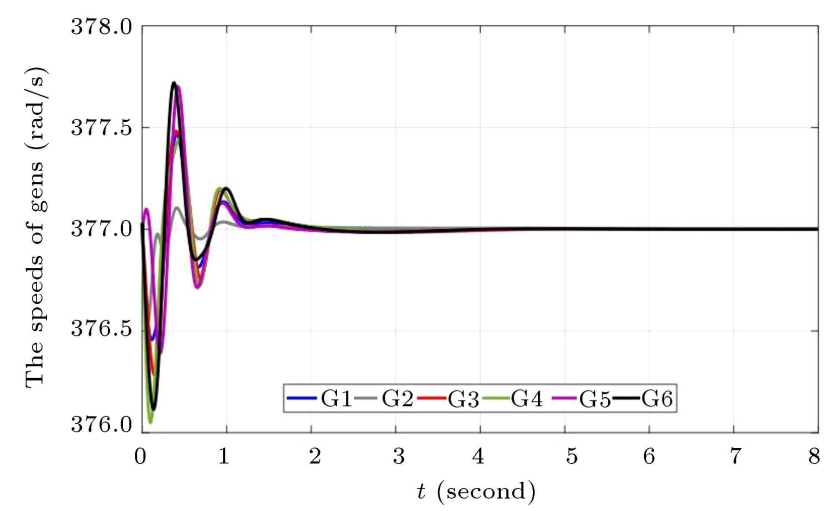

Figure 8. Rotor angular speeds of the synchronous machines with ASM controllers in Scenario 1.

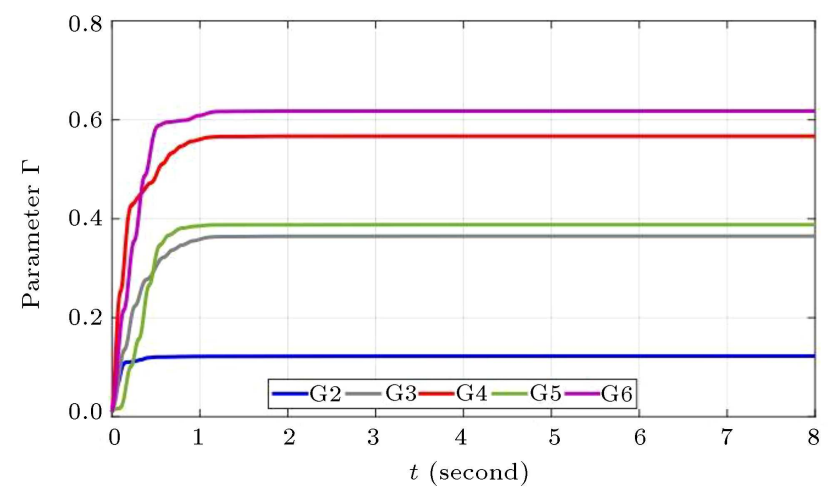

Figure 9. Adjustable gains $(\Gamma)$ for ASM controllers.
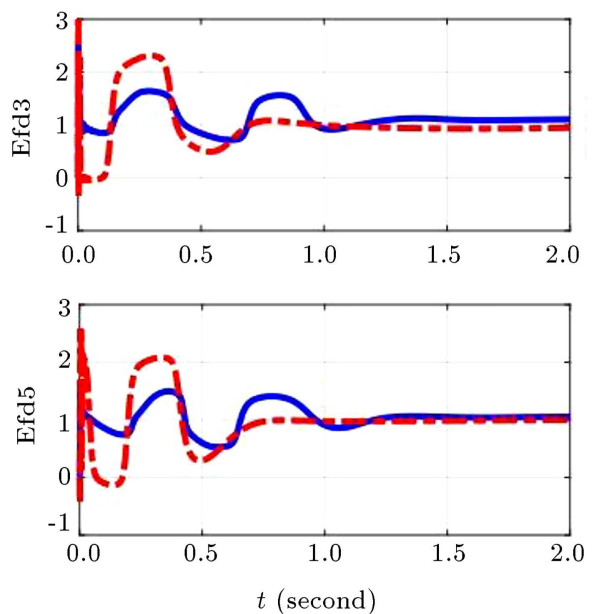

In this section, another study is conducted to compare the proposed controller with the CSM controller in terms of performance. The design of ASM and CSM controllers goes through the same stages, except that in the design of CSM controllers (considering Eq. (32)), $\Gamma$ is considered a numerical constant which is greater than the amount of the system's uncertain function such that Condition (33) is satisfied. The main limitation of the CSM controllers is the determination of the amount of the system's uncertain function, which is complex in practice.

Here, to compare the performance of the ASM and CSM controllers, the value of $\Gamma$ in CSM controllers is determined in two ways.

First, as shown in Figure 9, the maximum value of this parameter is related to the G6, which is 0.625 . It is assumed that G2-G6 are equipped with CSM controllers such that the value of $\Gamma$ in all of them is 0.625. Figure 10 compares the control signal $E_{f d}$ of G3-G6 in both cases.

As shown in Figure 10, the amplitude of the control signal $E_{f d}$ is higher when synchronous machines are equipped with CSM controllers. The lower amount of control signal $E_{f d}$ in the proposed ASM controller has the following benefits:

1. Lower control costs;

2. Reducing the probability of control blocks saturation.

Also, setting the maximum value for $\Gamma$ is a conservative choice and may result in increase in the probability of the occurrence of the chattering phenomenon.

Second, as shown in Figure 9, the minimum value of $\Gamma$ is related to $\mathrm{G} 2$, which is 0.1 . It is assumed that G2-G6 are equipped with CSM controllers and the value of $\Gamma$ in all of them is 0.1 . Figure 11 illustrates
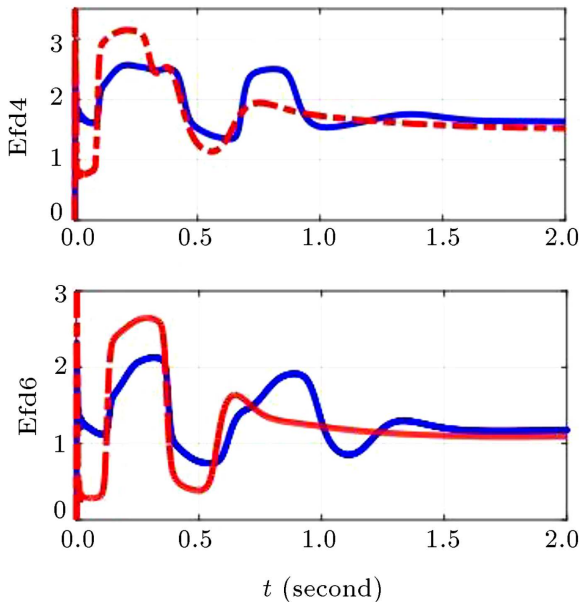

Figure 10. Control signal $E_{f d}$. Solid line: ASM controllers; dash-dotted line: CSM controllers. 


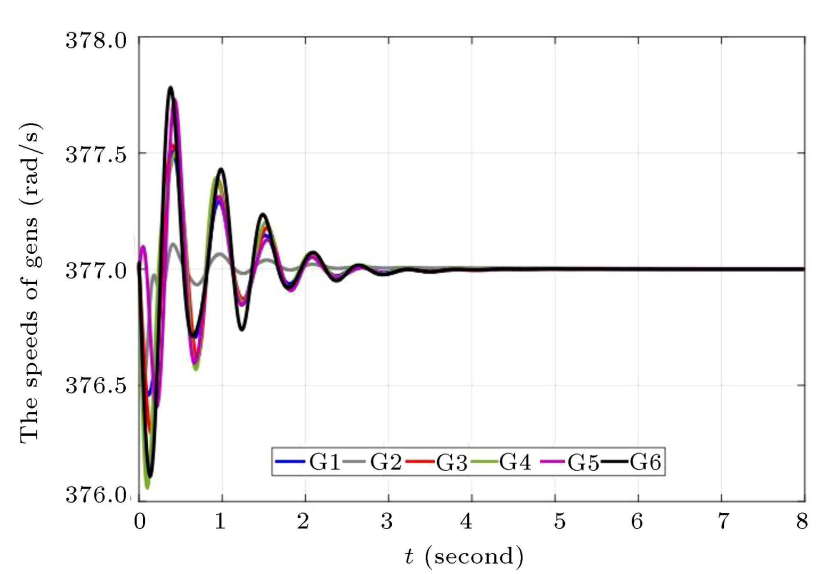

Figure 11. Rotor angular speeds of the synchronous machines with CSM controllers in Scenario 1.

the angular speeds of the synchronous machines in this case.

By comparing Figure 11 with Figure 8, it can be inferred that the settling time and amplitude of the oscillations have increased. Hence, the simulation results show the superiority of the ASM controllers to the CSM controllers. It should be noted that after stabilizing the system, the values of $\Gamma$ are reset to the initial values in ASM controllers.

\section{B. Scenario 2}

In this scenario, the performance of the proposed controller is evaluated in the face of structural and parametric uncertainties of the network. It is assumed that before $t=0 \mathrm{sec}$, the HVDC link is not in service and the network has been in a steady state condition. The structural uncertainties applied to the network are:

1. At $t=0 \mathrm{sec}$, the HVDC link is switched on;

2. At $t=3 \mathrm{sec}$, a $100 \mathrm{~ms}$ SLG fault is considered in the middle of the line 17-24. This fault is cleared at $t=3.1 \mathrm{sec}$ with line interruption and reclosing the line at $t=4.1 \mathrm{sec}$.

On the other hand, as parametric uncertainties, the following items are considered in the network:

1. An initial error is considered in the reactance of the transmission lines 1-22 and 2-23;

2. An initial error is considered in the direct axis transient reactance of the synchronous machines G3 and G6.

The initial errors of the mentioned parameters are considered in the range of $\pm 20 \%$ with a step of $5 \%$. Many simulations have been performed to ensure the correctness of the controller evaluation. In each simulation, the uncertainty of the above parameters is randomly selected and the simulation results are

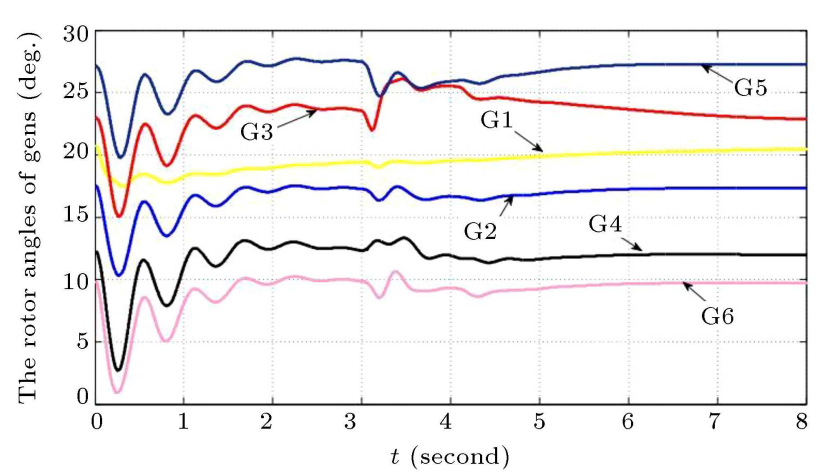

Figure 12. Rotor angles of the synchronous machines in Scenario 2.

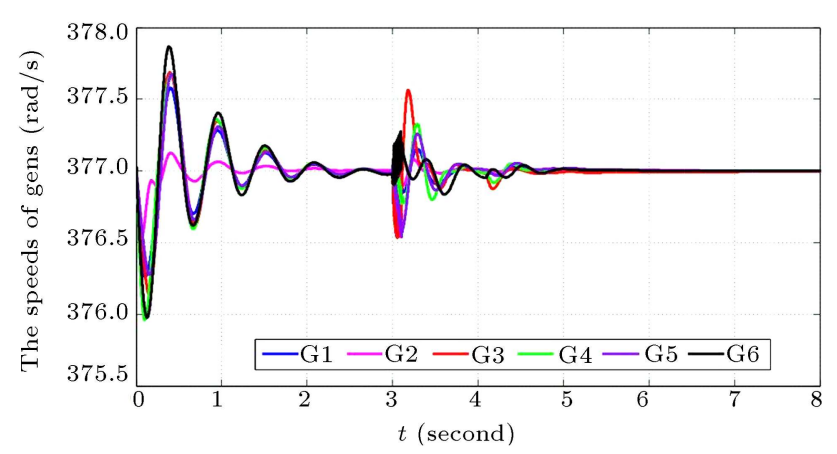

Figure 13. Rotor angular speeds of the synchronous machines in Scenario 2.

stored. At the end, the results of the simulations are averaged.

In this scenario, the HVDC-link controllers are adjusted to:

1. Injecting the desired active and reactive powers to the receiving end bus;

2. The buses of the both ends of the HVDC-link have the desired voltage magnitudes.

The process of analyzing the network during and after the fault has been performed according to the procedure described in Section 4. Figures 12 and 13 show the rotor angles and angular speeds of the synchronous machines, respectively, in this scenario.

Figure 14 shows the dampers and field windings currents for the synchronous Machine 3 as an example. This figure shows that during the fault-time, transient currents are generated in the damper windings, and when the fault is cleared, these currents converge to zero.

In this study, the HVDC link is modeled in detail. Figure 15 shows the voltage magnitude of the HVDC link on both sides of buses, the voltage of the DC link, and the injected active power to the receiving end of the HVDC link.

This figure illustrates the transients and dynamics of the HVDC-link waveforms in the face of system' parametric uncertainties and an asymmetrical fault. 

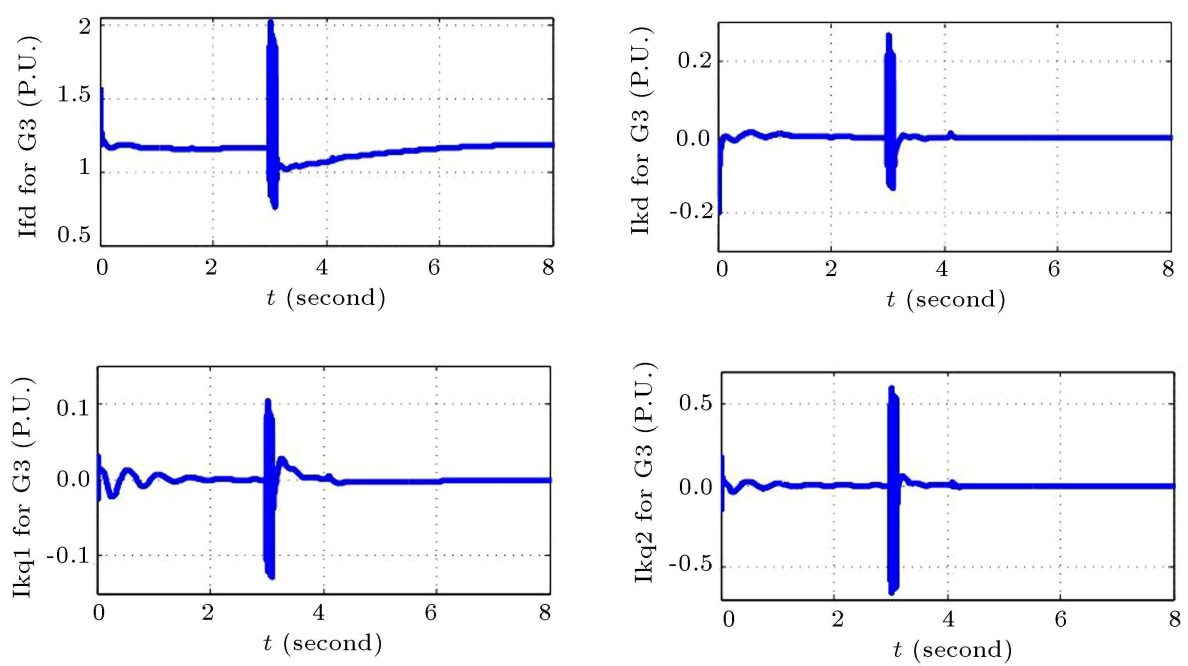

Figure 14. Currents of field and damper windings of the synchronous machine 3 in Scenario 2.
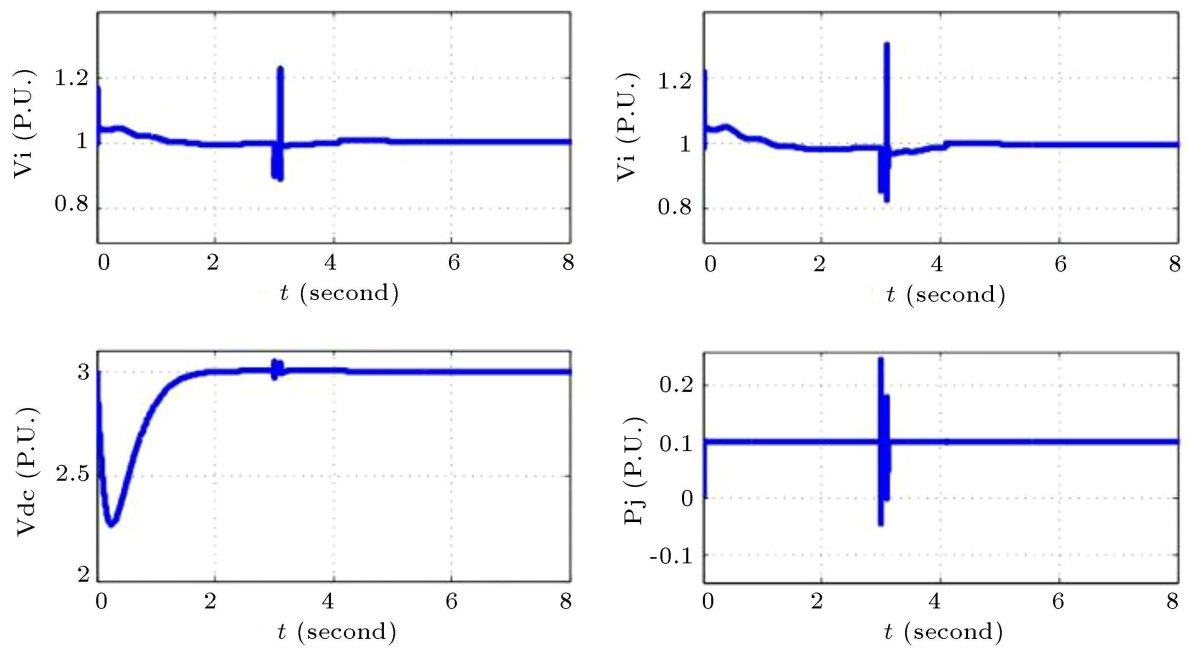

Figure 15. Waveforms related to HVDC in Scenario 2.

At last, Figure 16 shows the rotor angular speeds of the synchronous machines in Scenario 2 if, instead of an SLG fault, a Three-Line-to-Ground (TLG) fault is considered.

The simulation results in these scenarios indicate that the proposed controller improves the network stability under structural and parametric uncertainties.

\section{Conclusion}

In this paper, an ASM decentralized excitation controller was proposed to improve the stability of power systems under different uncertainties. Designing of the proposed controller was comparatively simple and effective and it required only local generator information. Simulation results on a multi-machine power system including an HVDC link show the satisfactory performance of the proposed controller. Also, the

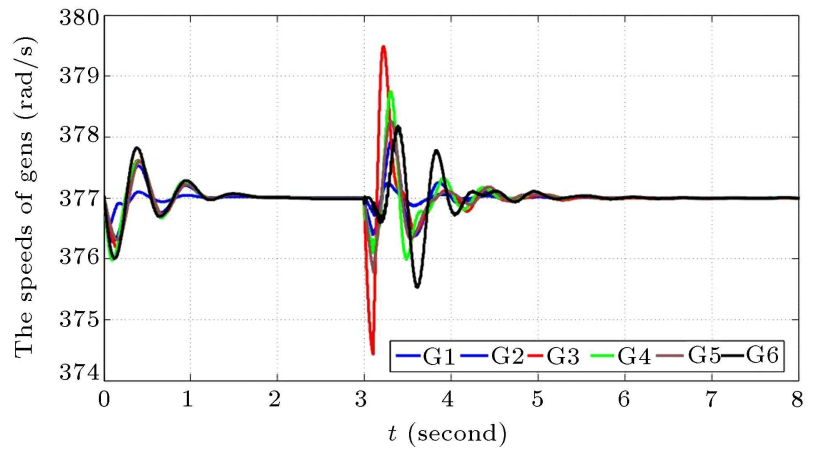

Figure 16. Rotor angular speeds of the synchronous machines in Scenario 2 for a TLG fault.

results demonstrate that the proposed controller needs lower control costs in comparing the CSM controller. Therefore, by using this controller, the probability of occurrence of the chattering phenomenon was reduced. 
Furthermore, in this paper, by using the space-phasor based sequence networks method, a procedure was proposed for dynamic analysis of power systems. The method is systematic such that it is simply possible to repeat the study of asymmetrical faults in different parts of the network by making minor changes to the admittance matrices of the sequence networks.

\section{References}

1. Kundur, P., Power System Stability and Control, 1st Edn., pp. 3-40, New York, McGraw-Hill (1994).

2. Chaturvedi, D.K., Malik, O.P., and Kalra, P.K. "Performance of a generalized neuron-based PSS in a multimachine power system", IEEE Transactions on Energy Conversion, 19(3), pp. 625-632 (2004).

3. Shojaeian, Sh., Soltani, J., and Arab Markadeh, Gh. "Damping of low frequency oscillations of multimachine multi-UPFC power systems, based on adaptive input-output feedback linearization control", IEEE Transactions on Power Systems, 27(4), pp. 1831-1840 (2012).

4. Preece, R., Milanovic, J.V., Almutairi, A.M., et al. "Probabilistic evaluation of damping controller in networks with multiple VSC-HVDC lines", IEEE Transactions on Power Systems, 28(1), pp. 367-376 (2013).

5. Knuppel, T., Nielsen, J.N., Jensen, K.H., et al. "Smallsignal stability of wind power system with full-load converter interfaced wind turbines", IET Renewable Power Generation, 6(2), pp. 79-91 (2012).

6. Vittal, V., McCalley, J.D., Anderson, P.M., et al., Power System Control and Stability, 3nd Edn., pp. 281-322, New York, Wiley-IEEE Press (2019).

7. Demello, F.P. and Concordia, C. "Concepts of synchronous machine stability as affected by excitation control", IEEE Transactions on Power Apparatus and Systems, PAS-88(4), pp. 316-329 (1969).

8. Dysko, A., Leithead, W.E., and O'Reilly, J. "Enhanced power system stability by coordinated PSS design", IEEE Transactions on Power Systems, 25(1), pp. 413422 (2010).

9. Kulkarni, N., Kamalasadan, S., and Ghosh, S. "An integrated method for optimal placement and tuning of a power system stabilizer based on full controllability index and generator participation", IEEE Transactions Industry Applications, 51(5), pp. 4201-4211 (2015).

10. Machowski, J., Robak, S., Bialek, J.W., et al. "Decentralized stability-enhancing control of synchronous generator", IEEE Transactions on Power Systems, 15(4), pp. 1336-1344 (2000).

11. Mahmud, M.A., Pota, H.R., Aldeen, M., et al. "Partial feedback linearizing excitation controller for multimachine power systems to improve transient stability", IEEE Transactions on Power Systems, 29(2), pp. 561-571 (2014).
12. Peric, V.S., Saric, A.T., and Grabez, D.I. "Coordinated tuning of power system stabilizers based on Fourier transform and neural networks", Electric Power Systems Research, 88, pp. 78-88 (2012).

13. Yousef, H. "Adaptive fuzzy logic load frequency control of multi-area power system", International Journal of Electrical Power \& Energy Systems, 68, pp. 384-395 (2015).

14. Utkin, V.I., Guldner, J., and Shi, J., Sliding Mode Control in Electromechanical Systems, pp. 1-13, London, U.K., Taylor \& Francis (1999).

15. Abidi, Kh. and Sabanovic, A. "Sliding-mode control for high-precision motion of Piezostage", IEEE Transactions on Industrial Electronics, 54(1), pp. 629-637 (2007)

16. Colbia-Vega, A., de Leon-Morales, J., Fridman, L., et al. "Robust excitation control design using slidingmode technique for multimachine power systems", Electric Power Systems Research, 78(9), pp. 16271634 (2008).

17. Ouassaid, M., Maaroufi, M., and Cherkaoui, M. "Observer-based nonlinear control of power system using sliding mode control strategy", Electric Power Systems Research, 84(1), pp. 135-143 (2012).

18. Huerta, H., Loukianov, A.G., and Canedo, J.M. "Multimachine power-system control: integral-SM approach", IEEE Transactions on Industrial Electronics, 56(6), pp. 2229-2236 (2009).

19. Huerta, H., Loukianov, A.G., and Canedo, J.M. "Decentralized sliding mode block control of multimachine power systems", International Journal of Electrical Power \& Energy Systems, 32(1), pp. 1-11 (2010).

20. Huerta, H., Loukianov, A.G., and Canedo, J.M. "Passivity-based sliding mode control of power systems", IEEE 10th International Workshop on Variable Structure Systems, pp. 343-348 (Jun. 2008).

21. Huerta, H., Loukianov, A.G., and Canedo, J.M. "Passivity sliding mode control of large-scale power systems", IEEE Transactions on Control Systems Technology, 27(3), pp. 1219-1227 (2019).

22. Lin, Z.W., Zhang, Z., Huang, H., et al. "Adaptive sliding mode control on single-machine infinite-bus power systems", IEEE Proceedings of the 31st Chinese Control Conference, pp. 3229-3234 (Jul. 2012).

23. Huang, Y.J., Kuo, T.C., and Chang, S.H. "Adaptive sliding-mode control for nonlinear systems with uncertain parameters", IEEE Transactions on Systems, Man, and Cybernetics, 38(2), pp. 534-539 (2008).

24. Samanfar, A., Shakarami, M.R., Soltani, J., et al. "Dynamic analysis of multi-machine multi-UPFC power systems experiencing transient asymmetrical faults", IET Generation, Transmission \& Distribution, 13(19), pp. 4491-4502 (2019).

25. Krause, P.C., Wasynczuk, O., Sudhoff, S.D., Analysis of Electric Machinery and Drive Systems, 2nd Edn., pp. 191-256, New York, Jhon Wiley \& Sons (2002). 
26. Lu, Q., Sun, Y., and Mei, Sh., Nonlinear Control Systems and Power System Dynamics, pp. 277-308, China, Kluwer Academic Publishers (2001).

\section{Biographies}

Amin Samanfar received his BSc degree in Electronic Engineering from Semnan University, Semnan, Iran in 2001 and his MSc degree from Tarbiat Modares University, Tehran, Iran in 2004. He is currently studying for his $\mathrm{PhD}$ degree at the Faculty of Technical \& Engineering, Lorestan University, Lorestan, Iran. His research interests are nonlinear control, power system control and dynamics, and flexible AC transmission systems.

Mahmoud Reza Shakarami was born in Khorramabad, Iran in 1972. He received his MS and PhD degrees in Electrical Engineering from Iran University of Science and Technology in Tehran, Iran in 2000 and 2009, respectively. He is an Associate Professor at the Department of Electrical Engineering, Lorestan University. His current research interests include power system dynamics and stability and FACTS Devices.

Jafar Soltani Zamani graduated from Tabriz Univer- sity, Tabriz, Iran in 1974 and received his MSc and PhD degrees from the University of Manchester Institute of Science and Technology, Manchester (U.M.I.S.T), U.K. in 1983 and 1987, respectively. He is currently a Professor in the Department of Electrical Engineering, Khomeinishar Branch, Islamic Azad University, Isfahan, Iran. He is also an Emeritus Professor at the Faculty of Electrical and Computer Engineering, Isfahan University of Technology, Isfahan, Iran. His main areas of research include electrical machines and drive, power electronics, and power system control. He has published many international journal and conference papers and is the holder of a U.K. patent. Dr. Soltani is a member of IEEE and IET. In addition, he is the reviewer of some international journals in European and IEEE Transactions.

Esmaeel Rokrok was born in Khorramabad, Iran in 1972. He received his $\mathrm{BSc}, \mathrm{MSc}$, and $\mathrm{PhD}$ degrees in Electrical Engineering from Isfahan University of Technology in 1995, 1997, and 2010, respectively. $\mathrm{He}$ is an Associate Professor at the Department of Electrical Engineering, Lorestan University. His major research interests lie in the area of power system control and dynamics, dispersed generation, microgrid, power electronic, and robust control. 